\title{
HARMONIC PERTURBATIONS \\ WITH DELAY OF PERIODIC SEPARATED VARIABLES DIFFERENTIAL EQUATIONS
}

\author{
Luca Bisconti — Marco Spadini
}

\begin{abstract}
We study the structure of the set of harmonic solutions to perturbed, nonautonomous, $T$-periodic, separated variables ODEs on manifolds. The perturbing term, supposed to be $T$-periodic in time, is allowed to contain a finite delay. Our main result extends those of [7] and [13] but it cannot be simply deduced from them: It emerges from of a combination of the techniques exposed in those two papers.
\end{abstract}

\section{Introduction}

In this paper we study harmonic solutions of $T$-periodic perturbations of $T$ periodic separated variables ODEs on manifolds, allowing the perturbing term to contain a finite delay. Namely, given $T>0, r \geq 0$ and a boundaryless smooth manifold $N \subseteq \mathbb{R}^{d}$, we consider $T$-periodic solutions of equations of the form

$$
\dot{\zeta}(t)=a(t) \Phi(\zeta(t))+\lambda \Xi(t, \zeta(t), \zeta(t-r)), \quad \lambda \geq 0,
$$

where $r>0$ is a finite time lag, $a: \mathbb{R} \rightarrow \mathbb{R}$ is a continuous $T$-periodic function, $\Phi: N \rightarrow \mathbb{R}^{d}$ and $\Xi: \mathbb{R} \times N \times N \rightarrow \mathbb{R}^{d}$ are given continuous tangent vector fields on $N$, in the sense that $\Phi(\xi)$ belongs to the tangent space $T_{\xi} N$, for any $\xi \in N$,

2010 Mathematics Subject Classification. 34K13, 34C40, 37C25, 34A09.

Key words and phrases. Delay differential equations, periodic solutions, differential equations on manifolds, translation operator, differential-algebraic equations. 
and $\Xi$ is $T$-periodic in the first variable and tangent to $N$ in the second one. That is,

$$
\Xi(t, \xi, \eta)=\Xi(t+T, \xi, \eta) \in T_{\xi} N, \quad \text { for all }(t, \xi, \eta) \in \mathbb{R} \times N \times N .
$$

We also assume that the average $\not$ of $a$ on the interval $[0, T]$ is nonzero, i.e.

$$
\not t:=\frac{1}{T} \int_{0}^{T} a(t) d t \neq 0 .
$$

Clearly, $a(t)$ can be written as $\not h+\alpha(t)$ where $\alpha: \mathbb{R} \rightarrow \mathbb{R}$ is continuous, $T$ periodic and with zero average. In this way, we get another interpretation of equation (1.1): we can regard it as the result of the introduction of a $T$-periodic perturbation with null average in the coefficient $\not k$ of the following equation:

$$
\dot{\zeta}(t)=\not \Phi(\zeta(t))+\lambda \Xi(t, \zeta(t), \zeta(t-r)), \quad \lambda \geq 0 .
$$

Our main objective is to provide information on the structure of the set of $T$-periodic solutions of (1.1) (recall that $T>0$ is given). Roughly speaking, we will give conditions ensuring the existence of a connected set of pairs $(\lambda, \zeta)$, with $\lambda \geq 0$ and $\zeta$ a $T$-periodic solution of (1.1) corresponding to $\lambda$, which are nontrivial (in the sense that either $\lambda>0$ or $\zeta$ is nonconstant), whose closure in an appropriate topological space is not compact and meets the set of pairs $(0, \zeta)$ with $\zeta$ a constant solution.

One may think that allowing the factor $a$ in (1.1) to be nonconstant would introduce only trivial alterations to the structure of $T$-periodic solutions of equation (1.3). It is not so. The following example shows how much such structure can change, even in the very simple circumstance of a perturbed scalar ODE:

Example 1.1. Consider the following parametrized scalar ordinary differential equation:

$$
\dot{x}(t)=a(t)(\sin (2 x)-x)+\lambda \sin (t), \quad \lambda \geq 0,
$$

for $x \in(-3,1.5)$. We examined three cases: (1) $a(t) \equiv 1 / 2$, (2) $a(t)=$ $\sin (t)+1 / 2$, and (3) $a(t)=\cos (t)+1 / 2$. We then computed numerically the initial conditions (for $t=0), x_{0}(\lambda)$, that lead to $2 \pi$-periodic solutions and plot them against $\lambda$. The resulting pictures are shown in Figure 1. As it is immediately seen, the set of $2 \pi$-periodic solutions can change dramatically for different factors $a$. Notice that all the considered $a$ 's share the same average $\not d=1 / 2$.

To pursue our goal we use topological tools as the fixed point index and the degree of tangent vector fields on manifolds (see, e.g. [5]). A deceptively natural approach would be to use a time transformation as in e.g. [13] and then to apply one of the known results for periodic perturbations of autonomous ODEs on manifolds as, for instance, those of [7]. However, this naive procedure does not work because the time-transformed perturbing term would result in a form 


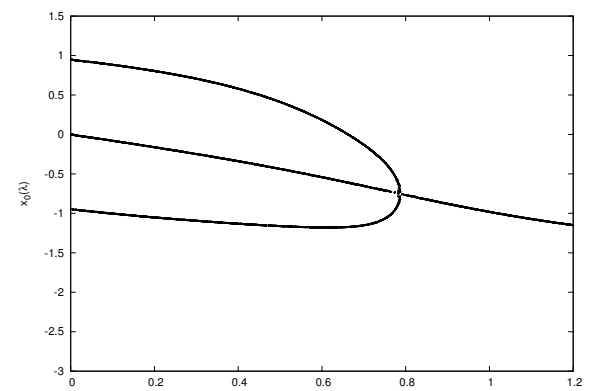

(a) The case $a(t) \equiv^{\lambda} 1 / 2, \lambda \in[0,1.2]$.

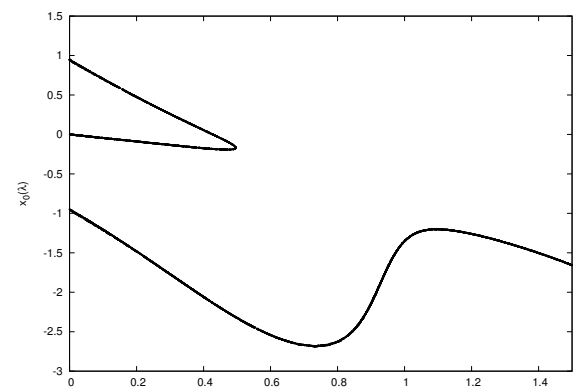

(b) The case $a(t)=\sin ^{\lambda}(t)+1 / 2, \lambda \in[0,1.5]$.

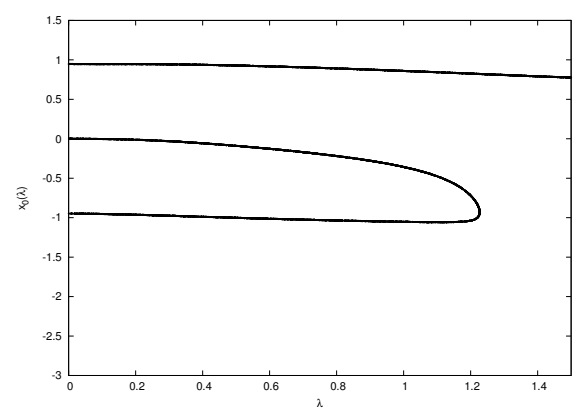

(c) The case $a(t)=\cos (t)+1 / 2, \lambda \in[0,1.5]$.

Figure 1. The set of $2 \pi$-periodic solutions for equation (1.4) for different choices of the factor $a$.

difficult to investigate (the reason is that the time-transformation of [13] does not preserve the fixed-delay structure). Our strategy, instead, consists of recasting and combining the arguments of [13] and [7]. To get a general idea of how we proceed, consider the particular case when $\Phi$ is $C^{1}$ (the general case when $\Phi$ is only continuous boils down to it via an approximation procedure). When $\alpha \equiv 0$ and the perturbation $\Xi$ does not depend on the delay, as in [6], our condition is obtained through a formula (see e.g. [5]) relating the degree of the tangent vector field $\Psi$ to the fixed point index of the translation operator at time $T, P_{T}^{\Phi}$, associated to the equation

$$
\dot{\zeta}=\Phi(\zeta)
$$

When $a$ is constant but the perturbing term in Equation (1.1) is allowed to contain a delay, as in [7], one needs to adapt this approach: the operator $P_{T}^{\Phi}$ is replaced with its infinite-dimensional analogue $Q_{T}^{\Phi}$ and the result is obtained through a formula that associates the degree of $\Phi$ to the fixed point index of $Q_{T}^{\Phi}$. In the present paper, in order to allow $a$ to be nonconstant, we revisit the construction in [7] and provide a relation (see Theorem 3.2 below) between the degree of $\Phi$ and the fixed point index of the infinite dimensional Poincaré type 
$T$-translation operator $Q_{T}^{a \Phi}$ associated to the separated variables equation $\dot{\zeta}=$ $a(t) \Phi(\zeta)$. Namely, $Q_{T}^{a \Phi}$ is the operator that associates to any element $\varphi \in$ $C([-r, 0], N)$ the function given by $\theta \mapsto \zeta(\varphi(0), \theta+T)$, with $\theta \in[-r, 0]$. Here $\zeta(p, \cdot)$ denotes the unique solution of the following Cauchy problem on $N$ :

$$
\dot{\zeta}=a(t) \Phi(\zeta), \quad \zeta(0)=p,
$$

and $C([-r, 0], N)$ is the subset of $C\left([-r, 0], \mathbb{R}^{d}\right)$ consisting of $N$-valued functions.

In order to illustrate our result, we conclude the paper with two "extended examples". The first concerns to the set of $T$-periodic solutions of a particular class of weakly coupled differential equations on manifolds. The second involves delay periodic perturbations of a family of semi-explicit differential-algebraic equations (DAEs).

The latter requires some further explanation. Generalizing [1], we study the structure of the set of $T$-periodic solutions of the following problem:

$$
\left\{\begin{array}{l}
\dot{x}(t)=a(t) f(x(t), y(t))+\lambda h(t, x(t), y(t), x(t-r), y(t-r)), \quad \lambda \geq 0 \\
g(x, y)=0
\end{array}\right.
$$

where $r>0$ is a finite time-lag, $f: U \rightarrow \mathbb{R}^{m}, h: \mathbb{R} \times U \times U \rightarrow \mathbb{R}^{m}$ and $g: U \rightarrow \mathbb{R}^{s}$ are given continuous maps defined on an open connected set $U \subseteq \mathbb{R}^{m} \times \mathbb{R}^{s} \cong \mathbb{R}^{d}$ and $h$ is $T$-periodic in the variable $t$. We also require that $g \in C^{\infty}\left(U, \mathbb{R}^{s}\right)$, with the property that the Jacobian matrix $\partial_{2} g(p, q)$ of $g$, with respect to the last $s$ variables, is invertible for any $(p, q) \in U$. Observe that this assumption implies that 0 is a regular value for $g$. So, $g^{-1}(0) \subseteq U$ is a closed $C^{\infty}$ submanifold of $\mathbb{R}^{m} \times \mathbb{R}^{s}$ of dimension $k$. Throughout the paper we will always denote the manifold $g^{-1}(0)$ by $M$; in this context, the points of $M$ will be written as pairs $(p, q)$. It is well-known (see e.g. [9]) that under these hypotheses it is always possible to transform the above DAE into an equivalent ODE of type (1.1) on the differentiable manifold $M$. Actually, as a direct consequence of the Implicit Function Theorem, $M$ can be locally represented as a graph of some map from an open subset of $\mathbb{R}^{m}$ to $\mathbb{R}^{s}$ and, hence Equation (1.5) can be locally decoupled. However, globally, this might be false or not convenient for our purpose (see e.g. [2]).

\section{Preliminaries and basic notions}

In this section we recall some basic facts and definitions about the function spaces used throughout the paper.

Let $I \subseteq \mathbb{R}$ be an interval and let $X \subseteq \mathbb{R}^{d}$. Given $r \in \mathbb{N} \cup\{0\}$, the set of all $X$-valued $C^{r}$-functions defined on $I$ is denoted by $C^{r}(I, X)$. When $I=\mathbb{R}$, we simply write $C^{r}(X)$ instead of $C^{r}(\mathbb{R}, X)$ and, when $r=0$, we simplify the notation writing $C(I, X)$ in place of $C^{0}(I, X)$ and $C(X)$ instead of $C^{0}(X)$. Let 
$T>0$ be given, by $C_{T}\left(\mathbb{R}^{d}\right)$ we mean the Banach space of all the continuous $T$-periodic functions $\zeta: \mathbb{R} \rightarrow \mathbb{R}^{d}$ whereas $C_{T}(X)$ denotes the metric subspace of $C_{T}\left(\mathbb{R}^{d}\right)$ consisting of all those $\zeta \in C_{T}\left(\mathbb{R}^{d}\right)$ that take values in $X$. It is not difficult to prove that $C_{T}(X)$ is complete if and only if $X$ is closed in $\mathbb{R}^{d}$.

Let $N \subseteq \mathbb{R}^{d}$ be a smooth differentiable manifold, and consider the following diagram of closed embeddings:

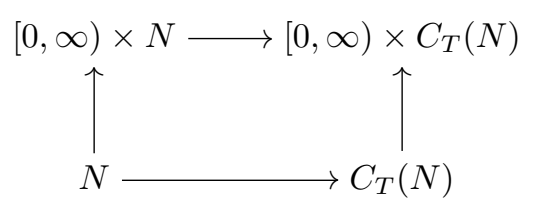

We identify any space in the above diagram with its image. In particular, $N$ will be regarded as its image in $C_{T}(N)$ under the embedding that associates to any $p \in N$ the function $\bar{p} \in C_{T}(N)$ constantly equal to $p$. Furthermore, we will regard $N$ as the slice $\{0\} \times N$ of $[0, \infty) \times N$ and, analogously, $C_{T}(N)$ as $\{0\} \times C_{T}(N)$. Thus, if $\Omega$ is a subset of $[0, \infty) \times C_{T}(N)$, then $\Omega \cap N$ represents the set of points of $N$ that, regarded as constant functions, belong to $\Omega$. Namely, with this convention, we have that

$$
\Omega \cap N=\{p \in N:(0, \bar{p}) \in \Omega\} .
$$

Let $\Theta: \mathbb{R} \times N \rightarrow \mathbb{R}^{d}$ be a time-dependent tangent vector field and assume that the Cauchy problem

$$
\left\{\begin{array}{l}
\dot{\zeta}=\Theta(t, \zeta), \\
\zeta(0)=p,
\end{array}\right.
$$

admits a unique solution for all $p \in N$. Denote by

$$
\mathcal{D}=\{(\tau, p) \in \mathbb{R} \times N \text { : the solution of (2.3) is continuable up to } t=\tau\} .
$$

A well known argument based on global continuation properties of the flows (see e.g. [10]) shows that $\mathcal{D}$ is an open set containing $\{0\} \times N$. Let $P^{\Theta}: \mathcal{D} \rightarrow N$ be the map that associates to each $(t, p) \in \mathcal{D}$ the value $\zeta(t)$ of the maximal solution $\zeta$ of (2.3), i.e. $P^{\Theta}(t, p)=\zeta(t)$. Here and in the sequel, given $\tau \in \mathbb{R}$, we denote by $P_{\tau}^{\Theta}=P^{\Theta}(\tau, \cdot)$ the (Poincaré) $\tau$-translation operator associated to problem (2.3). The domain $\mathcal{D}\left(P_{\tau}^{\Theta}\right)$ of $P_{\tau}^{\Theta}$ is an open (possibly empty) set formed by the points $p \in N$ for which the maximal solution of (2.3) is defined up to $\tau$. Clearly, $\mathcal{D}\left(P_{\tau}^{\Theta}\right)$ coincides with the slice $\mathcal{D}_{\tau}=\{p \in N:(\tau, p) \in \mathcal{D}\}$.

The remark below, borrowed from [13], plays a crucial role in what follows. 
REMARK 2.1. Let $\Phi: N \rightarrow \mathbb{R}^{d}$ as in (1.1). Consider the following Cauchy problems

$$
\begin{array}{llrl}
\dot{\zeta} & =\Phi(\zeta), & \zeta(0) & =\zeta_{0}, \\
\dot{\zeta} & =a(t) \Phi(\zeta), & \zeta(0) & =\zeta_{0},
\end{array}
$$

with $\zeta_{0} \in N$. Let $J$ and $I$ be the intervals on which are defined the (unique) maximal solutions of $(2.4 \mathrm{a})$ and $(2.4 \mathrm{~b})$ respectively. Suppose also that $\Phi$ is $C^{1}$, so that the uniqueness of solutions for the above problems is guaranteed. Let $u: I \rightarrow U$ and $\xi: J \rightarrow U$ be the maximal solutions of (2.4a) and (2.4b) respectively, with $I$ and $J$ the corresponding maximal intervals of existence.

Let $t>0$ be such that $\int_{0}^{l} a(s) d s \in I$ for all $l \in[0, t]$. Then it follows that

$$
\xi(t)=u\left(\int_{0}^{t} a(s) d s\right)
$$

and hence $t \in J$. Conversely, by a standard maximality argument, one can prove that $t \in J$ implies $\int_{0}^{t} a(s) d s \in I$. Assume that the average $\not h$ of $a$ is 1 , and define the map $\phi_{a}: J \rightarrow I, t \mapsto \phi_{a}(t)=\int_{0}^{t} a(s) d s$. Observe that $\phi$ is not necessarily invertible except when $a(t) \neq 0$ for all $t \in \mathbb{R}$. Notice also that, if $T \in J$, then $\phi_{a}(T)=T \in I$.

This remark has important consequences in terms of the $T$-translation (Poincaré) operators associated to the Cauchy problems (2.4a) and (2.4b). We collect them in the following proposition.

Proposition 2.2. Let $P_{T}^{\Phi}$ and $P_{T}^{a \Phi}$ be the T-translation operators associated to the Cauchy problems $(2.4 \mathrm{a})$ and $(2.4 \mathrm{~b})$, respectively. Then $P_{T}^{a \Phi}\left(\zeta_{0}\right)$ is defined if and only if so is $P_{T}^{\Phi}\left(\zeta_{0}\right)$. If we assume in addition that the average of a on $[0, T]$ is equal to 1 , we have $P_{T}^{\Phi}\left(\zeta_{0}\right)=P_{T}^{a \Phi}\left(\zeta_{0}\right)$, whenever $P_{T}^{a \Phi}$ or $P_{T}^{\Phi}$ is defined.

Proof. It follows immediately from Remark 2.1.

The following fact is an immediate consequence of the above result (see also [13, Corollary 2.4]).

Proposition 2.3. Let $\Phi: N \rightarrow \mathbb{R}^{k}$ be a $C^{1}$ tangent vector field, and let $a: \mathbb{R} \rightarrow \mathbb{R}$ be continuous and $T$-periodic with $\not \alpha=1$. Given an open subset $V$ of $N$, if $\operatorname{ind}\left(P_{T}^{a \Phi}, V\right)$ is well defined, then so is $\operatorname{ind}\left(P_{T}^{\Phi}, V\right)$ and

$$
\operatorname{ind}\left(P_{T}^{a \Phi}, V\right)=\operatorname{ind}\left(P_{T}^{\Phi}, V\right)=\operatorname{deg}(-\Phi, V) .
$$

The symbols "ind" and "deg" that appear in the above theorem denote, respectively, the fixed point index of a map and the degree of a tangent vector field. Roughly speaking, the former counts (algebraically) the fixed points of a map, and the latter counts the zeros of a vector field. For an exposition of this topic we refer, e.g. to [5], [8], [11]. 
We conclude this section with an useful fact that we will use later in some examples.

Let $U \subseteq \mathbb{R}^{m} \times \mathbb{R}^{s}=: \mathbb{R}^{k}$ be open and connected. Let $g: U \rightarrow \mathbb{R}^{s}$ be a $C^{\infty}$ map such that the the partial derivative, $\partial_{2} g(x, y)$, of $g$ with respect to the second $s$-variable is invertible for each $(x, y) \in U$. Then, $M=g^{-1}(0)$ is a smooth manifold in $\mathbb{R}^{m} \times \mathbb{R}^{s}$.

The following theorem holds (see [2, Theorem 4.1] and [14, Theorem 4.1]):

TheOREM 2.4. Let $M$ be as above and let $\Phi: M \rightarrow \mathbb{R}^{m} \times \mathbb{R}^{s}$ be a tangent vector field. Define $F: \mathbb{R}^{k} \times \mathbb{R}^{s} \rightarrow \mathbb{R}^{k} \times \mathbb{R}^{s}$ by

$$
F(p, q):=\left(\widehat{\Phi}_{1}(p, q), g(p, q)\right),
$$

where $\widehat{\Phi}_{1}$ is the first $\mathbb{R}^{m}$-component of any continuous extension $\widehat{\Phi}$ of $\Phi$ to $U$. Then, for any $V \subseteq U$ open, if either $\operatorname{deg}(\Phi, N \cap V)$ or $\operatorname{deg}(F, V)$ is well defined, so is the other, and

$$
|\operatorname{deg}(\Phi, N \cap V)|=|\operatorname{deg}(F, V)| .
$$

Observe that an extension of $\Phi$, as in the above theorem, always exists by the well known Tietze's Theorem (see, e.g., [3]).

The right-hand-side of $(2.7)$ is, in principle, easier to compute than $\operatorname{deg}(\Phi$, $N \cap V$ ) because $F$ is defined on the open subset $V$ of the "flat" space $\mathbb{R}^{m} \times \mathbb{R}^{s}$ rather than on the "curved" set $N \cap V$, as it is the case with $\Phi$. In fact, when $V$ is relatively compact and $F$ is nonzero on its boundary, $\operatorname{deg}(F, V)$ coincides with the Brouwer degree of $F$ (seen as a self-map of $\mathbb{R}^{m} \times \mathbb{R}^{s}$ ) in $V$ relative to 0 .

\section{Infinite-dimensional Poincaré-type translation operator}

Let $N \subseteq \mathbb{R}^{d}$ be as above, and let $\Phi: N \rightarrow \mathbb{R}^{d}$ and $\Xi: \mathbb{R} \times N \times N \rightarrow \mathbb{R}^{d}$ be continuous tangent vector fields on $N$. Given $T>0$, assume also that $\Xi$ is $T$-periodic in $t$. Consider the delay differential equation (1.1) where, we recall, $r>0$ and $a: \mathbb{R} \rightarrow \mathbb{R}$ is continuous, $T$-periodic and with average $\not k=(1 / T) \int_{0}^{T} a(t) d t \neq 0$. We are interested in the $T$-periodic solutions of (1.1). Without loss of generality we can assume that $T \geq r$ (see e.g. [6]). In fact, for $n \in \mathbb{N}$, Equation (1.1) and

$$
\dot{\zeta}(t)=a(t) \Phi(\zeta(t))+\lambda \Xi(t, \zeta(t), \zeta(t-(r-n T))), \quad \lambda \geq 0,
$$

share the same $T$-periodic solutions (although other solutions may be quite different). Thus, if necessary, one can replace $r$ with $r-n T$, where $n \in N$ is chosen such that $0<r-n T \leq T$.

Let us now establish some further notation. For a given $T>0$ and $X \subseteq \mathbb{R}^{d}$, $\widetilde{X}$ denotes the metric space $\widetilde{X}:=C([-T, 0], X)$ with the distance inherited from the Banach space $\widetilde{\mathbb{R}^{n}}=C\left([-T, 0], \mathbb{R}^{n}\right)$ with the usual supremum norm. Observe that $\widetilde{X}$ is complete if and only if $X$ is closed in $\mathbb{R}^{n}$. Given any $p \in N$, denote 
by $\widetilde{p} \in \widetilde{N}$ the constant function $\widetilde{p}(t) \equiv p, t \in[-T, 0]$. Moreover, for any $V \subseteq N$, and $W \subseteq \widetilde{N}$ we define the sets

$$
V^{\#}:=\{\widetilde{p} \in \widetilde{N}: p \in V\} \quad \text { and } \quad W_{\#}:=\{p \in N: \widetilde{p} \in W\} .
$$

Notice also that, for any given $V \subseteq N$, one has $V^{\#} \subseteq \widetilde{V}$ and $(\widetilde{V})_{\#}=V$.

Proceeding as in $[6, \S 3]$, we now introduce a Poincaré-type $T$-translation operator on an open subset of $\widetilde{N}$. Here, we assume that $\Phi$ is $C^{1}$. Let $Q_{T}^{\Phi}$ be the map defined, whatever $\phi \in \widetilde{N}$ it makes sense for, by

$$
Q_{T}^{\Phi}(\phi)(\theta)=\zeta(\phi(0), T+\theta), \quad \theta \in[-r, 0],
$$

where $\zeta(p, \cdot)$ denotes the unique maximal solution of the Cauchy problem

$$
\begin{aligned}
\dot{\zeta}(t) & =\Phi(\zeta(t)), \\
\zeta(0) & =p .
\end{aligned}
$$

Well known properties of differential equations imply that the domain $\mathcal{D}\left(Q_{T}^{\Phi}\right)$ of $Q_{T}^{\Phi}$ is an open subset of $\widetilde{N}$. Moreover, since $T \geq r$, the Ascoli-Arzelà Theorem implies that $Q_{T}^{\Phi}$ is a locally compact map (see e.g. [12]). Observe that there is a simple relation between $\mathcal{D}\left(Q_{T}^{\Phi}\right)$ and $\mathcal{D}\left(P_{T}^{\Phi}\right)$, where $P_{T}^{\Phi}$ is the translation operator defined in Section 2. Namely,

$$
\mathcal{D}\left(Q_{T}^{\Phi}\right)=\left\{\varphi \in \widetilde{N}: \varphi(0) \in \mathcal{D}\left(P_{T}^{\Phi}\right)\right\} .
$$

In particular, $\widetilde{\mathcal{D}\left(P_{T}^{\Phi}\right)} \subseteq \mathcal{D}\left(Q_{T}^{\Phi}\right)$. Notice also that $P_{T}^{\Phi}(p)=Q_{T}^{\Phi}(\widetilde{p})(0)$ for all $p \in \mathcal{D}\left(P_{T}^{\Phi}\right)$. Similarly, given $a: \mathbb{R} \rightarrow \mathbb{R}$ as above, we define the map $Q_{T}^{a \Phi}$ by setting, for whatever $\phi \in \widetilde{N}$ it makes sense,

$$
Q_{T}^{a \Phi}(\phi)(\theta)=\xi(\phi(0), T+\theta), \quad \theta \in[-r, 0],
$$

where $\xi(p, \cdot)$ is the unique maximal solution of the Cauchy problem

$$
\begin{aligned}
\dot{\xi}(t) & =a(t) \Phi(\xi(t)), \\
\xi(0) & =p .
\end{aligned}
$$

Since $\mathcal{D}\left(P_{T}^{\Phi}\right)=\mathcal{D}\left(P_{T}^{a \Phi}\right)$, it follows easily that $\mathcal{D}\left(Q_{T}^{\Phi}\right)=\mathcal{D}\left(Q_{T}^{a \Phi}\right)$.

It is not difficult to prove that the $T$-periodic solutions of (3.1a) are in a one-to-one correspondence with the fixed points of $Q_{T}^{\Phi}$. Similarly, the $T$-periodic solutions of (3.2a) are in a one-to-one correspondence with the fixed points of $Q_{T}^{a \Phi}$. Moreover, if $\not k=1$, Proposition 2.2 implies that the fixed points of $P_{T}^{\Phi}$ coincide with those of $P_{T}^{a \Phi}$. However, even in this case, $Q_{T}^{\Phi}$ might be different from $Q_{T}^{a \Phi}$. We wish to obtain a formula for the fixed point index of admissible pairs $\left(Q_{T}^{a \Phi}, W\right)$, with $W$ open in $\mathcal{D}\left(Q_{T}^{a \Phi}\right)$. Observe, in fact, that the same argument used for $Q_{T}^{\Phi}$ shows that $Q_{T}^{a \Phi}$ is locally compact when $T \geq r$. In the case when $a(t) \equiv 1$, we have the following result ([7, Theorem 3.2]). 
THEOREM 3.1. Let $\Phi$ be as above and let $W \subseteq \tilde{N}$ be open and such that $\operatorname{ind}\left(Q_{T}^{\Phi}, W\right)$ is defined. Then, $\operatorname{deg}\left(-\Phi, W_{\#}\right)$ is defined as well and

$$
\operatorname{ind}\left(Q_{T}^{\Phi}, W\right)=\operatorname{deg}\left(-\Phi, W_{\#}\right) .
$$

It is not difficult to see that, for any constant $c$ and any tangent vector field $v$, admissible on an open $V \subseteq N$, one has

$$
\operatorname{deg}(c v, V)=(\operatorname{sign} c)^{\operatorname{dim} N} \operatorname{deg}(v, V) .
$$

Hence, when $a(t) \equiv \not h$, Equation (3.3) yields

$$
\operatorname{ind}\left(Q_{T}^{\not \Phi \Phi}, W\right)=\operatorname{deg}\left(-\not L \Phi, W_{\#}\right)=(\operatorname{sign} \not h)^{\operatorname{dim} N} \operatorname{deg}\left(-\Phi, W_{\#}\right) .
$$

We seek to generalize this formula to the case when $a$ is nonconstant. The first part of our construction is inspired by the proof of Theorem 3.2 in [7]; there are a few essential difference, though, due to the presence of the factor $a$. These are located, mostly, in the second part of the proof.

Theorem 3.2. Let $a, \Phi$ and $T$ be as in (1.1). Assume that $\Phi$ is $C^{1}$ and let $Q_{T}^{a \Phi}$ be as above. Let also $W \subseteq \tilde{N}$ be open. If the fixed point index $\operatorname{ind}\left(Q_{T}^{a \Phi}, W\right)$ is defined, then so is $\operatorname{deg}\left(\Phi, W_{\#}\right)$ and

$$
\operatorname{ind}\left(Q_{T}^{a \Phi}, W\right)=(\operatorname{sign} \not h)^{\operatorname{dim} N} \operatorname{deg}\left(-\Phi, W_{\#}\right) .
$$

In particular, one has that

$$
\operatorname{ind}\left(Q_{T}^{a \Phi}, W\right)=(\operatorname{sign} \not h)^{\operatorname{dim} N} \operatorname{ind}\left(Q_{T}^{\Phi}, W\right) .
$$

Proof. The assumption that $\operatorname{ind}\left(Q_{T}^{a \Phi}, W\right)$ is defined means that $W \subseteq$ $\mathcal{D}\left(Q_{T}^{a \Phi}\right)$ and that the fixed point set $\operatorname{Fix}\left(Q_{T}^{a \Phi}\right) \cap W$ is compact. Let us show that $\operatorname{deg}\left(\Phi, W_{\#}\right)$ is defined too. We need to prove that $\Phi^{-1}(0) \cap W_{\#}$ is compact. If $p \in \Phi^{-1}(0) \cap W_{\#}$, then the constant function $\widetilde{p}$ is clearly a fixed point of $Q_{T}^{a \Phi}$. Thus $\Phi^{-1}(0) \cap W_{\#}$ is compact since it can be regarded as a closed subset of $\operatorname{Fix}\left(Q_{T}^{a \Phi}\right) \cap W$.

We now use the Commutativity Property of the fixed point index in order to obtain a relation between the indices of $P_{T}^{a \Phi}$ and $Q_{T}^{a \Phi}$. Define the maps $h: \mathcal{D}\left(P_{T}^{a \Phi}\right) \rightarrow \widetilde{N}$ and $k: \widetilde{N} \rightarrow N$ by $h(p)(\theta)=\xi(p, \theta+T)$ and $k(\phi)=\phi(0)$, respectively. Here, $\xi(p, \cdot)$ indicates the unique maximal solution of the Cauchy problem (3.2). One has that

$(3.7 \mathrm{a})(h \circ k)(\phi)(\theta)=\xi(\phi(0), \theta+T)=Q_{T}^{a \Phi}(\phi)(\theta), \quad \phi \in \mathcal{D}\left(Q_{T}^{a \Phi}\right), \theta \in[-r, 0]$,

$$
(k \circ h)(p)=\left.\xi(p, \theta+T)\right|_{\theta=0}=\xi(p, T)=P_{T}^{a \Phi}(p), \quad p \in \mathcal{D}\left(P_{T}^{a \Phi}\right) .
$$

Define $\gamma=\left.k\right|_{W}$. As a consequence of the Commutativity Property of the fixed point index, $\operatorname{ind}\left(h \circ \gamma, \gamma^{-1}\left(\mathcal{D}\left(P_{T}^{a \Phi}\right)\right)\right)$ is defined if and only if $\operatorname{ind}\left(\gamma \circ h, h^{-1}(W)\right)$ is defined as well and, in this case,

$$
\operatorname{ind}\left(h \circ \gamma, \gamma^{-1}\left(\mathcal{D}\left(P_{T}^{a \Phi}\right)\right)\right)=\operatorname{ind}\left(\gamma \circ h, h^{-1}(W)\right) .
$$


Moreover, since $W \subseteq \mathcal{D}\left(Q_{T}^{a \Phi}\right)$, then $\gamma^{-1}\left(\mathcal{D}\left(P_{T}^{a \Phi}\right)\right)=W$. Hence, from (3.7), it follows that

$$
\begin{aligned}
\operatorname{ind}\left(Q_{T}^{a \Phi}, W\right) & =\operatorname{ind}\left(h \circ \gamma, \gamma^{-1}\left(\mathcal{D}\left(P_{T}^{a \Phi}\right)\right)\right), \\
\operatorname{ind}\left(P_{T}^{a \Phi}, h^{-1}(W)\right) & =\operatorname{ind}\left(\gamma \circ h, h^{-1}(W)\right) .
\end{aligned}
$$

Recall that, according to Remark 2.1, $\mathcal{D}\left(P_{T}^{\Phi}\right)=\mathcal{D}\left(P_{T}^{a \Phi}\right)$ so that $h^{-1}(W) \subseteq$ $\mathcal{D}\left(P_{T}^{\Phi}\right)$. Then by $(3.8)$ and $(3.9)$ we get

$$
\operatorname{ind}\left(Q_{T}^{a \Phi}, W\right)=\operatorname{ind}\left(P_{T}^{a \Phi}, h^{-1}(W)\right) .
$$

Assume now, in addition, that the average $\not$ of $a$ is equal to 1. Proposition 2.3 yields

$$
\operatorname{ind}\left(P_{T}^{a \Phi}, h^{-1}(W)\right)=\operatorname{ind}\left(P_{T}^{\Phi}, h^{-1}(W)\right)=\operatorname{deg}\left(-\Phi, h^{-1}(W)\right) .
$$

By the definition of $h$, one has that $\Phi^{-1}(0) \cap W_{\#}=\Phi^{-1}(0) \cap h^{-1}(W)$. In fact, all the constant solutions of $(3.2 \mathrm{a})$ lie in $W_{\#}$. Then, from the Excision Property of the degree of a tangent vector field, we get

$$
\operatorname{deg}\left(-\Phi, h^{-1}(W)\right)=\left(-\Phi, W_{\#}\right) .
$$

Therefore, we get (3.5) by (3.10)-(3.12).

Let us now remove the additional assumption on $a$. Let us put $a_{0}(t)=a(t) / \not d$ for all $t \in \mathbb{R}$ and $\Phi_{a}(p)=\not \phi \Phi(p)$ for all $p \in N$. We rewrite equation (3.2) as follows:

$$
\dot{\xi}(t)=a_{0}(t) \Phi_{a}(\xi(t)),
$$

and observe that $Q_{T}^{a_{0} \Phi_{a}}=Q_{T}^{a \Phi}$. Since the average of $a_{0}$ over $[0, T]$ is equal to 1 , using the first part of the proof, we get that

$$
\operatorname{ind}\left(Q_{T}^{a \Phi}, W\right)=\operatorname{ind}\left(Q_{T}^{a_{0} \Phi_{a}}, W\right)=\operatorname{deg}\left(-\Phi_{a}, W_{\#}\right)=\operatorname{deg}\left(-\not h \Phi, W_{\#}\right) .
$$

Since from (3.4) we have

$$
\operatorname{deg}\left(-\not \iota \Phi, W_{\#}\right)=(\operatorname{sign} \not h)^{\operatorname{dim} N} \operatorname{deg}\left(-\Phi, W_{\#}\right),
$$

the assertion follows from (3.13).

\section{Branches of starting pairs for (1.1)}

Any pair $(\lambda, \varphi) \in[0, \infty) \times \widetilde{N}$ is said to be a starting pair for (1.1) if the following initial value problem has a $T$-periodic solution:

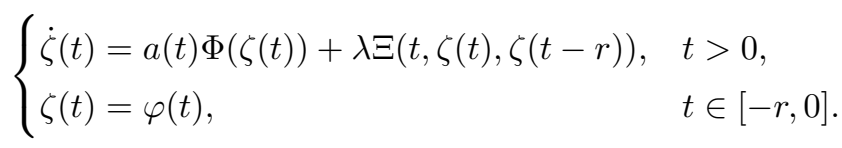

A pair of the type $(0, \widetilde{p})$ with $\Phi(p)=0$ is clearly a starting pair and will be called a trivial starting pair. The set of all starting pairs for (1.1) will be denoted by $S$. For the reminder of this section we assume that $\Phi$ and $\Xi$ are $C^{1}$, so 
that (4.1) admits a unique maximal solution that we denote by $\xi^{\lambda}(\varphi, \cdot)$. By known continuous dependence properties of delay differential equations the set $\mathcal{V} \subseteq[0, \infty) \times \tilde{N}$ given by

$$
\mathcal{V}:=\left\{(\lambda, \varphi) \in[0, \infty) \times \widetilde{N}: \xi^{\lambda}(\varphi, \cdot) \text { is defined on }[0, T]\right\}
$$

is open. Clearly $\mathcal{V}$ contains the set $S$ of all starting pairs for (1.1). Observe that $S$ is closed in $\mathcal{V}$, even if it may be not so in $[0,+\infty) \times \widetilde{N}$. Moreover, by the Ascoli-Arzelà Theorem it follows that $S$ is locally compact.

It is convenient to introduce the following notation for the "slices" of product spaces. Let $Y$ be a set. Given $X \subseteq[0, \infty) \times Y$, we put $X_{\lambda}:=\{\varphi \in Y:(\lambda, \varphi) \in$ $X\}$ for each $\lambda \geq 0$.

Theorem 4.1 below concerns connected set of starting pairs for (1.1). It will play a key role in our main result. Its proof, based on a global connectivity result [4, Lemma 1.4] and Theorem 3.2 above, follows with some adaptations along the lines of Section 4 in [7].

Theorem 4.1. Assume that $\Phi, \Xi, S$ are as above and let $a: \mathbb{R} \rightarrow \mathbb{R}$ be continuous and T-periodic such that its average on a period is nonzero. Let $W \subseteq[0, \infty) \times \widetilde{N}$ be open. If $\operatorname{deg}\left(\Phi,\left(W_{0}\right)_{\#}\right)$ is defined and nonzero, then the set

$$
(S \cap W) \backslash\{(0, \widetilde{p}) \in W: \Phi(p)=0\}
$$

of nontrivial starting pairs in $W$ admits a connected subset whose closure in $S \cap W$ meets $\{(0, \widetilde{p}) \in W: \Phi(p)=0\}$ and is not compact.

Proof. Consider the open set $U=W \cap \mathcal{V}$. Since $\Phi^{-1}(0) \cap\left(U_{0}\right)_{\#}=\Phi^{-1}(0) \cap$ $\left(W_{0}\right)_{\#}$, and $S \cap U=S \cap W$, we need to prove that the set of nontrivial starting pairs in $U$ admits a connected subset whose closure in $S \cap U$ meets the set $\{(0, \widetilde{p}) \in U: \Phi(p)=0\}$ and is not compact. Consider the the pair of topological spaces

$$
(Y, Z)=\left(S \cap U,\left\{(0, \widetilde{p}) \in U: p \in \Phi^{-1}(0)\right\}\right) .
$$

From Lemma 1.4 of [4] applied to $(Y, Z)$ follows that if all compact subsets of $Y$ containing $Z$ have nonempty boundary, then $Y \backslash Z$ contains a connected set $\Gamma$ whose closure (in $Y$ ) intersects $Z$ and is not compact. In fact, the closure of $\Gamma$ satisfies all the requirements of the theorem.

Since $U$ is open and $S$ is locally compact $S \cap U$ is locally compact too. Moreover, the assumption that $\operatorname{deg}\left(\Phi,\left(W_{0}\right)_{\#}\right)$ is defined means that the set

$$
\left\{p \in\left(W_{0}\right)_{\#}: \Phi(p)=0\right\}=\left\{p \in\left(U_{0}\right)_{\#}: \Phi(p)=0\right\}
$$

is compact. Thus the homeomorphic set $\{(0, \widetilde{p}) \in U: \Phi(p)=0\}$ is compact as well. Let us now prove that there are no compact subsets of $S \cap U$ containing $Z$ and with empty boundary in $S \cap U$. 
We will argue by contradiction. Assume that there exists such a set $C$. Then $C$ is relatively open in $S \cap U$ and $(S \cap U) \backslash C$ is closed in $S \cap U$. Hence, the distance $\delta=\operatorname{dist}(C,(S \cap U) \backslash C)$ between the compact set $C$ and $(S \cap U) \backslash C$ is nonzero. Define the map $\mathcal{Q}: \mathcal{V} \rightarrow \widetilde{N}, \mathcal{V}$ being as in (4.2), given by

$$
\mathcal{Q}(\lambda, \varphi)(\theta)=\xi^{\lambda}(\varphi, \theta+T), \quad \theta \in[-r, 0],
$$

where $\xi^{\lambda}(\varphi, \cdot)$ is the unique maximal solution of (4.1). Notice that $\mathcal{Q}(0, \cdot)$ coincides with the map $Q_{T}^{a \Phi}$ defined in the previous section. In fact, if $\zeta(p, \cdot)$ is the unique solution of the Cauchy problem $(3.2)$, then we have $\xi^{0}(\varphi(0), \cdot)=$ $\zeta(\varphi(0), \cdot)$.

Observe that $\mathcal{Q}$ is continuous by well-known results on delay differential equations, and it is not difficult to prove that $\mathcal{Q}$ is a locally compact map. Consider the set

$$
A=\{(\lambda, \varphi) \in U: \operatorname{dist}((\lambda, \varphi), C)<\delta / 2\},
$$

which, clearly, does not meet $(S \cap U) \backslash C$. The compactness of $S \cap U \cap A=C$ imply that for some $\lambda_{*}>0$ one has $\left(\left\{\lambda_{*}\right\} \times A_{\lambda_{*}}\right) \cap S \cap U=\emptyset$. So, since the set $\{(\lambda, \varphi) \in A: \mathcal{Q}(\lambda, \varphi)=\varphi\}$ is compact being coincident with $S \cap U \cap A$, the Generalized Homotopy Invariance Property of the fixed point index imply that

$$
0=\operatorname{ind}\left(\mathcal{Q}\left(\lambda_{*}, \cdot\right), A_{\lambda_{*}}\right)=\operatorname{ind}\left(\mathcal{Q}(0, \cdot), A_{0}\right),
$$

Thus, as $Q_{T}^{a \Phi}=\mathcal{Q}(0, \cdot)$, Theorem 3.2 and the Excision Property of the degree yield

$$
0=\operatorname{ind}\left(\mathcal{Q}(0, \cdot), A_{0}\right)=\operatorname{ind}\left(Q_{T}^{a \Phi}, A_{0}\right)=\left|\operatorname{deg}\left(\Phi,\left(A_{0}\right)_{\#}\right)\right|=\left|\operatorname{deg}\left(\Phi,\left(W_{0}\right)_{\#}\right)\right|,
$$

contradicting the hypothesis of the theorem.

\section{Branches of $T$-periodic pairs for (1.1)}

A pair $(\lambda, \zeta) \in[0, \infty) \times C_{T}(N)$, where $\zeta$ a is $T$-periodic solution of (1.1), is called a $T$-periodic pair. Those $T$-periodic pairs that are of the particular form $(0, \bar{p})$ are said to be trivial. Here and in the sequel, as introduced in (2.1), $\bar{p} \in C_{T}(N)$ denotes the function, defined on $\mathbb{R}$, identically equal to $p \in N$. Clearly $\widetilde{p}=\left.\bar{p}\right|_{[-T, 0]}$, where $\widetilde{p}$ is the function introduced in the previous section. Notice that, since $a$ is not identically zero, $(0, \bar{p}) \in[0, \infty) \times C_{T}(N)$ is a trivial $T$-periodic pair if and only if $\Phi(p)=0$. We point out that if $\zeta$ is a nonconstant $T$-periodic solution of the unperturbed equation $\dot{\zeta}=a(t) \Phi(\zeta)$, then $(0, \zeta)$ is a nontrivial $T$-periodic pair. Recall that $\Phi: N \rightarrow \mathbb{R}^{d}, \Xi: \mathbb{R} \times N \times N \rightarrow \mathbb{R}^{d}$ and $a: \mathbb{R} \rightarrow \mathbb{R}$ are continuous with $\Phi$ and $\Xi$ tangent to $N$ in the sense specified in the Introduction. We also assume that $a$ and $\Xi$ are $T$-periodic in $t$ and $a$ has nonzero average on a period. 
In this section we focus on the $T$-periodic solutions to (1.1). In fact, we study the topological structure of the set of pairs $(\lambda, x) \in[0, \infty) \times C_{T}(N)$ where $x$ is a solution of this equation. Our result extends that of $[7, \S 5]$.

The following is our main result. Its proof, based on Theorem 4.1 above, is not too far from that of [7, Theorem 5.1] (see also [4]). For this reason we only provide a sketch. In fact, here, we try to clarify the argument by a more schematic approach.

Theorem 5.1. Let $a, \Phi$ and $\Xi$ as above. Let $\Omega \subseteq[0, \infty) \times C_{T}(N)$ be open and such that $\operatorname{deg}(\Phi, \Omega \cap N)$ is defined and nonzero. Then $\Omega$ contains a connected set of nontrivial T-periodic pairs for (1.1) whose closure in $\Omega$ meets the set $\{(0, \bar{p}) \in \Omega: \Phi(p)=0\}$ and is not compact. In particular, the set of T-periodic pairs for (1.1) contains a connected component that meets $\{(0, \bar{p}) \in \Omega: \Phi(p)=0\}$ and whose intersection with $\Omega$ is not compact.

The following lemma takes care of the special case when the vector fields in (1.1) are $C^{1}$. The proof of the theorem will then be completed by the means of an approximation procedure.

Lemma 5.2. Let a, $\Phi, \Xi$ and $\Omega$ be as in Theorem 5.1. Assume in addition that $\Phi$ and $\Xi$ are $C^{1}$. Then $\Omega$ contains a connected set of nontrivial $T$-periodic pairs for (1.1) whose closure in $\Omega$ meets the set $\{(0, \bar{p}) \in \Omega: \Phi(p)=0\}$ and is not compact.

Proof (sketch). (Follows the first part of [7, Theorem 5.1].) Denote by $X$ the topological space of $T$-periodic pairs of (1.1) and by $S$ the one of starting pairs of the same equation. Define the map $h: X \rightarrow S$ by $h(\lambda, \zeta)=\left(\lambda,\left.\zeta\right|_{[-r, 0]}\right)$. It is readily shown that our assumptions on $\Phi$ and $\Xi$ imply that $h$ is a homeomorphism. Consider the set

$$
S_{\Omega}=\{(\lambda, \varphi) \in S: \text { the solution of (1.1) is contained in } \Omega\},
$$

so that $X \cap \Omega$ and $S_{\Omega}$ correspond under $h$. Thus, $S_{\Omega}$ being an open subset of $S$, we can find an open subset $W$ of $[0, \infty) \times \widetilde{N}$ such that $S \cap W=S_{\Omega}$. It is not difficult to show that the set $\left\{p \in\left(W_{0}\right)_{\#}: \Phi(p)=0\right\}$ coincides with $\{p \in \Omega \cap N: \Phi(p)=0\}$, so that the excision property of the degree gives $\operatorname{deg}\left(\Phi,\left(W_{0}\right)_{\#}\right)=\operatorname{deg}(\Phi, \Omega \cap N) \neq 0$. Theorem 4.1, yields a connected set $\Sigma \subseteq$ $(S \cap W) \backslash\{(0, \widetilde{p}) \in W: \Phi(p)=0\}$ whose closure in $S \cap W$ meets $\{(0, \widetilde{p}) \in W$ : $\Phi(p)=0\}$ and is not compact.

The set $\Gamma=h^{-1}(\Sigma) \subseteq X \cap \Omega$ is a connected set of nontrivial $T$-periodic pairs whose closure in $X \cap \Omega$ meets $\{(0, \bar{p}) \in \Omega: \Phi(p)=0\}$ and is not compact. Now, $X$ being closed in $[0, \infty) \times C_{T}(N)$, the closures of $\Gamma$ in $X \cap \Omega$ and in $\Omega$ coincide. Therefore $\Gamma$ satisfies the requirements.

The proof of Theorem 5.1 can now be performed by approximation. 
Proof (Sketch of the Proof of Theorem 5.1). Let us now prove the first part of the assertion. As in the last part of the proof of Lemma 5.2, it is enough to show the existence of a connected set $\Gamma$ of nontrivial $T$-periodic pairs whose closure in $X \cap \Omega$ meets $\{(0, \bar{p}) \in \Omega: \Phi(p)=0\}$ and is not compact.

Observe that the closed subset $X$ of $[0, \infty) \times C_{T}(N)$ is locally compact because of Ascoli-Arzelà Theorem. It is convenient to introduce the following subset of $X$ :

$$
\Upsilon=\left\{(0, \bar{p}) \in[0, \infty) \times C_{T}(N): \Phi(p)=0\right\} .
$$

Take $Y=X \cap \Omega$ and $Z=\Upsilon \cap \Omega$ and notice that $Y$ is locally compact as an open subset of $X$. Moreover, $Z$ is a compact subset of $Y$ (recall that, by assumption, $\operatorname{deg}(\Psi, N \cap \Omega)$ is defined). Since $Y$ is closed in $\Omega$, we can proceed as in Proposition 4.1 and deduce the assertion from Lemma 1.4 of [4] applied to the pair $(Y, Z)$. In order to do so we need to prove that the assumptions of Lemma 1.4 of [4] are satisfied. We argue by contradiction assuming that there exists a relatively open compact subset $C$ of $Y$ containing $Z$. For all $0<\rho<\operatorname{dist}(C, Y \backslash C)$, we let $A^{\rho}$ be the set of all pairs $(\lambda, \varphi) \in \Omega$ whose distance from $C$ is smaller than $\rho$. Thus, $A^{\rho} \cap Y=C$ and $\partial A^{\rho} \cap Y=\emptyset$. It is not difficult to prove that $\rho$ can be chosen in such a way that $A^{\rho}$ is bounded with complete closure, $A^{\rho} \cap N$ is a relatively compact subset of $\Omega \cap N$, and $\Phi$ is nonzero on the boundary (relative to $N$ ) of $A^{\rho} \cap N$.

Known approximation results on manifolds yield sequences $\left\{\Phi_{i}\right\}_{i \in \mathbb{N}}$ and $\left\{\Xi_{i}\right\}_{i \in \mathbb{N}}$ of $C^{1}$ tangent vector fields on $N$ uniformly approximating $\Phi$ and $\Xi$, respectively, with $T$-periodic $\Xi_{i}$ in the first variable. Thus, for $i \in \mathbb{N}$ large enough, we get

$$
\operatorname{deg}\left(\Phi_{i}, A^{\rho} \cap N\right)=\operatorname{deg}\left(\Phi, A^{\rho} \cap N\right)=\operatorname{deg}(\Phi, \Omega \cap N) \neq 0
$$

(the second equality follows by the excision property of the degree). Lemma 5.2 applied to the equation

$$
\dot{x}(t)=a(t) \Phi_{i}(x(t))+\lambda \Xi_{i}(t, x(t), x(t-r)),
$$

yields a connected subset $\Gamma_{i}$ of $A^{\rho}$ whose closure in $A^{\rho}$ meets $\Upsilon_{i} \cap A^{\rho}$ and is not compact. Here, $\Upsilon_{i}=\left\{(0, \bar{p}) \in[0, \infty) \times C_{T}(N): \Phi_{i}(p)=0\right\}$.

Let us denote by $\bar{\Gamma}_{i}$ and $\overline{A^{\rho}}$ the closures in $[0, \infty) \times C_{T}(N)$ of $\Gamma_{i}$ and $A^{\rho}$, respectively. It is not difficult to prove that, for sufficiently large $i$ 's, $\bar{\Gamma}_{i}$ is compact. One can also prove, as a consequence of this fact, that $\bar{\Gamma}_{i} \cap \partial A^{\rho} \neq \emptyset$ when $i$ is large enough.

Let $X_{i}$ denote the set of $T$-periodic pairs of (5.1). Clearly, $X_{i}$ being closed, $\bar{\Gamma}_{i} \subseteq X_{i}$. This implies the existence of a $T$-periodic pair $\left(\lambda_{i}, x_{i}\right) \in \partial A^{\rho}$ of (5.1).

By Ascoli-Arzelà Theorem, we may assume that, as $i \rightarrow \infty, x_{i} \rightarrow x_{0}$ in $C_{T}(N)$ and $\lambda_{i} \rightarrow \lambda_{0}$ with $\left(\lambda_{0}, x_{0}\right) \in \partial A^{\rho}$. Passing to the limit in Equation (5.1), it is not difficult to show that $\left(\lambda_{0}, x_{0}\right)$ is a $T$-periodic pair for (1.1) in $\partial A^{\rho}$. This 
is a contradiction, because by construction $\partial A^{\rho} \cap Y=\emptyset$. We have shown that the assumptions of Lemma 1.4 of [4] are satisfied. So, as anticipated, the first part of the assertion is proved.

Let us prove the last part of the thesis. Consider the connected component $\Xi$ of $X$ that contains the connected set $\Gamma$ of the first part of the assertion. We show that $\Xi$ has the required properties. Clearly, $\Xi$ meets the set $\{(0, \bar{p}) \in \Omega$ : $\Phi(p)=0\}$ because so does the closure of $\Gamma$ in $\Omega$. Moreover, $\Xi \cap \Omega$ cannot be compact, since $\Xi \cap \Omega$, as a closed subset of $\Omega$, contains the closure of $\Gamma$ in $\Omega$, which is not compact. This completes the proof.

In view of Theorem 2.4, one has that Theorem 5.1 assumes a somewhat nicer form when the manifold is defined implicitly. Instead of stating this fact formally in a corollary, we illustrate it with the following simple example:

EXAmple 5.3. Consider the map $g: \mathbb{R}^{2} \times \mathbb{R} \rightarrow \mathbb{R}$ defined by $g\left(\left(x_{1}, x_{2}\right), y\right)=$ $y^{3}+y-x_{1}^{2}-x_{2}^{2}$, and let $N=g^{-1}(0)$. Let $f: \mathbb{R}^{2} \times \mathbb{R} \rightarrow \mathbb{R}^{3}$ be given by $f\left(\left(x_{1}, x_{2}\right), y\right)=\left(x_{2},-x_{1}, 0\right)$. A simple computation shows that the restriction $\Phi$ of $f$ to $N$ is a tangent vector field. Also, take $T=1, a(t)=|\sin (2 \pi t)|$ and $\Omega=[0, \infty) \times C_{T}(N)$. As in (2.6), we define the map $F: \mathbb{R}^{2} \times \mathbb{R} \rightarrow \mathbb{R}^{3}$, by $F\left(\left(x_{1}, x_{2}\right), y\right)=\left(x_{2},-x_{1}, y^{3}+y-x_{1}^{2}-x_{2}^{2}\right)$. Using Theorem 2.4 we have that

$$
|\operatorname{deg}(\Phi, \Omega \cap N)|=\left|\operatorname{deg}\left(F, \mathbb{R}^{3}\right)\right|=\left|\operatorname{deg}_{B}\left(F, \mathbb{R}^{3}, 0\right)\right|=1,
$$

where " $\operatorname{deg}_{B}$ " denotes the Brouwer degree. Hence $\operatorname{deg}(\Phi, \Omega \cap N) \neq 0$ and the assertion of Theorem 5.1 holds for any $T$-periodic tangent vector field $\Xi: \mathbb{R} \times$ $N \times N \rightarrow \mathbb{R}^{3}$.

\section{Examples}

The purpose of this section is to illustrate the techniques developed in the foregoing ones. In order to do so, we examine here two classes of separated variables perturbed systems.

6.1. Weakly coupled equations. Here, we consider delay periodic perturbations of a particular family of ordinary differential equations on product manifolds. Namely, if $N_{1} \subseteq \mathbb{R}^{n_{1}}$ and $N_{2} \subseteq \mathbb{R}^{n_{2}}$ are boundaryless smooth manifolds, we consider the following differential equation on $N=N_{1} \times N_{2}$ :

$$
\left\{\begin{array}{l}
\dot{x}_{1}(t)=a_{1}(t) \Phi_{1}\left(x_{1}(t)\right)+\lambda \Xi_{1}\left(t, x_{1}(t), x_{2}(t), x_{1}(t-r), x_{2}(t-r)\right), \\
\dot{x}_{2}(t)=a_{2}(t) \Phi_{2}\left(x_{2}(t)\right)+\lambda \Xi_{2}\left(t, x_{1}(t), x_{2}(t), x_{1}(t-r), x_{2}(t-r)\right),
\end{array}\right.
$$

where, for $i=1,2, \Phi_{i}: N_{i} \rightarrow \mathbb{R}^{n_{i}}$ and $\Xi_{i}: \mathbb{R} \times N \times N \rightarrow \mathbb{R}^{n_{i}}$ are continuous tangent vector fields to $N_{i}$ with respect to the variable $x_{i}$. We also assume that $\Xi_{i}$ and the maps $a_{i}: \mathbb{R} \rightarrow \mathbb{R}$ are continuous, $T$-periodic in $t$, for $i=1,2$, and that the average $\phi_{i}$ of $a_{i}$ on a period is nonzero. 
Clearly, when $\lambda=0$, the resulting unperturbed equations are completely decoupled. In essence, the perturbation provides the (only) coupling in (6.1).

Actually, Theorem 5.1 cannot be applied directly to (6.1). However, we can use the same strategy. We only sketch the argument here. As in Remark 2.1, assume that, for $i=1,2, \Phi_{i}$ are $C^{1}$ so that uniqueness of the solutions of the following initial value problems on $N=N_{1} \times N_{2}$ hold:

$$
\left\{\begin{array}{l}
\dot{x}_{1}=\Phi_{1}\left(x_{1}\right), \\
\dot{x}_{2}=\Phi_{2}\left(x_{2}\right), \\
x(0)=\left(x_{1}(0), x_{2}(0)\right)=\xi^{0}=\left(\xi_{1}^{0}, \xi_{2}^{0}\right),
\end{array}\right.
$$

and

$$
\left\{\begin{array}{l}
\dot{x}_{1}=a_{1}(t) \Phi_{1}\left(x_{1}\right), \\
\dot{x}_{2}=a_{2}(t) \Phi_{2}\left(x_{2}\right), \\
x(0)=\left(x_{1}(0), x_{2}(0)\right)=\xi^{0}=\left(\xi_{1}^{0}, \xi_{2}^{0}\right),
\end{array}\right.
$$

and let $u: I \rightarrow N$ and $\xi: J \rightarrow N$ be the maximal solutions of (6.2a) and (6.2b), respectively, with $I$ and $J$ the relative maximal intervals of existence. Let $t>0$ be such that $\int_{0}^{l} a_{i}(s) d s \in I, i=1,2$, for all $l \in[0, t]$, then it follows that

$$
\xi(t)=\left(\xi_{1}(t), \xi_{2}(t)\right)=\left(u_{1}\left(\int_{0}^{t} a_{1}(s) d s\right), u_{2}\left(\int_{0}^{t} a_{2}(s) d s\right)\right),
$$

and hence $t \in J$. Conversely, by a maximality argument, it can be shown that $T \in J$ implies $\int_{0}^{t} a_{i}(s) d s \in I, i=1,2$.

As in Section 3 we construct an "infinite dimensional" $T$-translation operator associated to (6.1) for $\lambda=0$. Namely, we let $Q_{T}^{a_{1}, a_{2}}$ be the map that to any $\varphi \in \tilde{N}$ associates the map $\theta \mapsto \zeta(\varphi(0), T+\theta)$, whenever it makes sense to do so. Here $\zeta(p, \cdot)$ denotes the unique solution of $(6.2 \mathrm{a})$ with $\xi^{0}=\varphi(0)$.

Let $\Phi: N \rightarrow \mathbb{R}^{n_{1}} \times \mathbb{R}^{n_{2}}=\mathbb{R}^{n_{1}+n_{2}}$ be the tangent vector field on $N$ given by $\Phi\left(p_{1}, p_{2}\right)=\left(\Phi_{1}\left(p_{1}\right), \Phi_{2}\left(p_{2}\right)\right)$. The following result similar to Theorem 3.2 holds:

Proposition 6.1. Let $a_{1}, a_{2}, \Phi_{1}, \Phi_{2}, N_{1}, N_{2}, N, T$ and $Q^{a_{1}, a_{2}}$ be as above. Take $W \subseteq \widetilde{N}$ open and such that the fixed point index of $Q^{a_{1}, a_{2}}$ is defined in $W$, then so is $\operatorname{deg}\left(\Phi, W_{\#}\right)$ and

$$
\operatorname{ind}\left(Q^{a_{1}, a_{2}}, W\right)=\left(\operatorname{sign} \not \phi_{1}\right)^{\operatorname{dim} N_{1}}\left(\operatorname{sign} \not \phi_{2}\right)^{\operatorname{dim} N_{2}} \operatorname{deg}\left(\Phi, W_{\#}\right) .
$$

Proof (sketch). The assertion can be proved by following closely the argument of Theorem 3.2 and taking into account the following well-known and easily verified fact of degree theory:

For any given pair of constants $c_{1}, c_{2} \in \mathbb{R} \backslash\{0\}$ and tangent vector fields $v_{1}: N_{1} \rightarrow \mathbb{R}^{n_{1}}$ and $v_{2}: N_{2} \rightarrow \mathbb{R}^{n_{2}}$, admissible on an open $\mathcal{V} \subseteq N$, one has

$$
\operatorname{deg}\left(-v_{c}, \mathcal{V}\right)=\left(-\operatorname{sign} c_{1}\right)^{\operatorname{dim} N_{1}}\left(-\operatorname{sign} c_{2}\right)^{\operatorname{dim} N_{2}} \operatorname{deg}(v, \mathcal{V}) .
$$


where $v, v_{c}: N \rightarrow \mathbb{R}^{n_{1}+n_{2}}$ are the tangent vector fields on $N$ given by $v\left(p_{1}, p_{2}\right)=$ $\left(v_{1}\left(p_{1}\right), v_{2}\left(p_{2}\right)\right)$ and $v_{c}\left(p_{1}, p_{2}\right)=\left(c_{1} v_{1}\left(p_{1}\right), c_{2} v_{2}\left(p_{2}\right)\right)$ for all $\left(p_{1}, p_{2}\right) \in N$.

Any $(\lambda, \zeta) \in[0, \infty) \times C_{T}(N)$, with $\zeta$ solution of $(6.1)$, is a $T$-periodic pair. Such a pair is trivial if $\lambda=0$ and $\zeta$ is constant. An argument that follows very closely the one of Theorem 5.1 yields the following result:

Proposition 6.2. For $i=1,2$, let $\Phi_{i}: N_{i} \rightarrow \mathbb{R}^{n_{i}}$, be (continuous) tangent vector fields, and let $\Xi_{i}: \mathbb{R} \times N \times N \rightarrow \mathbb{R}^{n_{i}}$ be tangent to $N_{i}$ in the second variable; assume that the $\Xi_{i}$ 's as well as the maps $a_{i}: \mathbb{R} \rightarrow \mathbb{R}$, are continuous, $T$-periodic in $t$. Suppose also that, for $i=1,2$, the average of $\phi_{i}$ of $a_{i}$ is nonzero. Let $\Omega \subseteq[0, \infty) \times C_{T}(N)$ be open, and assume that $\operatorname{deg}(\Phi, \Omega \cap N)$ is defined and nonzero. Then $\Omega$ contains a connected set of nontrivial T-periodic pairs of (6.1) whose closure in $\Omega$ meets the set $\{(0, \bar{p}) \in \Omega: \Phi(p)=(0)\}$ and is not compact.

Example 6.3. Let $T=2 \pi$ and consider the following system of equations in $\mathbb{R}^{3}$

$$
\left\{\begin{array}{l}
\dot{x}_{2}=(2+\sin (t))\left(x_{2}+x_{3}\right) \\
\dot{x}_{1}-\dot{x}_{3}=|\cos (t)| x_{1}-(2+\sin (t)) x_{3} \\
-\dot{x}_{2}+\dot{x}_{3}=-(2+\sin (t)) x_{2}
\end{array}\right.
$$

that we write more compactly as follows:

$$
E \dot{x}=A(t) x
$$

where, for $t \in \mathbb{R}$,

$$
E=\left(\begin{array}{rrr}
0 & 1 & 0 \\
1 & 0 & -1 \\
0 & -1 & 1
\end{array}\right) \quad \text { and } \quad A(t)=\left(\begin{array}{ccc}
0 & 2+\sin (t) & 2+\sin (t) \\
|\cos (t)| & 0 & -2-\sin (t) \\
0 & -2-\sin (t) & 0
\end{array}\right)
$$

Let us now consider the following $2 \pi$-periodic perturbation of (6.4):

$$
E \dot{x}(t)=A(t) x(t)+\lambda \mathcal{H}(t, x(t), x(t-r)), \quad \lambda \geq 0,
$$

where $r>0$ is a given time lag and $\mathcal{H}: \mathbb{R} \times \mathbb{R}^{3} \times \mathbb{R}^{3} \rightarrow \mathbb{R}^{3}$ is a continuous map which is $2 \pi$-periodic in its first variable. Multiplying (6.5) on the left by $E^{-1}$ and setting, for all $(t, p, q) \in \mathbb{R} \times \mathbb{R}^{3} \times \mathbb{R}^{3}$,

$$
H(t, p, q)=E^{-1} \mathcal{H}(t, p, q) \quad \text { and } \quad B(t)=E^{-1} A(t)
$$

we see that (6.5) becomes

$$
\dot{x}(t)=B(t) x(t)+\lambda H(t, x(t), x(t-r)) .
$$

Clearly,

$$
B(t)=\left(\begin{array}{ccc}
|\cos (t)| & 0 & 0 \\
0 & 2+\sin (t) & 0 \\
0 & 0 & 2+\sin (t)
\end{array}\right)\left(\begin{array}{lll}
1 & 0 & 0 \\
0 & 1 & 1 \\
0 & 0 & 1
\end{array}\right)
$$


Hence, identifying $\mathbb{R}^{3}$ with the product space $\mathbb{R} \times \mathbb{R}^{2}$, we see that (6.6) falls into the family of weakly coupled systems (6.1) with $N_{1}=\mathbb{R}$ and $N_{2}=\mathbb{R}^{2}$. Let $\Phi: \mathbb{R} \times \mathbb{R}^{2} \rightarrow \mathbb{R}^{3}$ be given by $\Phi\left(p ; q_{1}, q_{2}\right):=\left(p, q_{1}+q_{2}, q_{2}\right)$, and take $\Omega=$ $[0, \infty) \times C_{T}\left(\mathbb{R}^{3}\right)$. Since, $\Phi$ is admissible for the degree in $\Omega \cap \mathbb{R}^{3}=\mathbb{R}^{3}$ and $\operatorname{deg}\left(\Phi, \mathbb{R}^{3}\right)=1$, by Proposition 6.2, there exists a connected set of nontrivial $T$-periodic pairs of (6.6) whose closure meets the set $\Phi^{-1}(0)=\{(0 ; 0,0)\}$ and is not compact. Since solutions of (6.6) are solutions of (6.5) and vice versa, this statement concerns, in fact, the $T$-periodic solutions of (6.5).

6.2. Periodic solutions of a class of DAEs of type (1.5). Here, as in the Introduction, $U \subseteq \mathbb{R}^{k} \times \mathbb{R}^{s}$ is open and connected and $g: U \rightarrow \mathbb{R}^{s}$ is $C^{\infty}$ with the property that $\partial_{2} g(x, y)$ is nonsingular for any $(x, y) \in U$. In this way, $M=g^{-1}(0)$ is a $C^{\infty}$ manifold of $\mathbb{R}^{k} \times \mathbb{R}^{s}$. We also require that $f$ and $h$, as in Equation (1.5), are continuous and that $a$ and $h$ are $T$-periodic in $t$ with the average of $a$ different from zero.

In what follows, we say that $(\lambda,(x, y)) \in[0, \infty) \times C_{T}(U)$ is a $T$-periodic pair of $(1.5)$, if $(x, y)$ is a $T$-periodic solution of (1.5) corresponding to $\lambda$. According to the convention introduced in (2.1)-(2.2), any $(p, q) \in U$ is identified with the element $(\bar{p}, \bar{q})$ of $C_{T}(U)$ that is constantly equal to $(p, q)$. A $T$-periodic pair of the form $(0,(\bar{p}, \bar{q}))$ will be called trivial. This subsection is devoted to the study of the set of $T$-periodic pairs of Equation (1.5).

Because of our assumption on $g$ it is possible to associate tangent vector fields on $M$ to the functions $f$ and $h$ in (1.5). Consider first maps $\Psi: U \rightarrow \mathbb{R}^{k} \times \mathbb{R}^{s}$ and $\Upsilon: \mathbb{R} \times U \times U \rightarrow \mathbb{R}^{k} \times \mathbb{R}^{s}$ as follows:

$$
\begin{aligned}
& \Psi\left(p_{1}, q_{1}\right)=\left(f\left(p_{1}, q_{1}\right),-\left[\partial_{2} g\left(p_{1}, q_{1}\right)\right]^{-1} \partial_{1} g\left(p_{1}, q_{1}\right) f\left(p_{1}, q_{1}\right)\right), \\
& \Upsilon\left(t,\left(p_{1}, q_{1}\right),\left(p_{2}, q_{2}\right)\right)= \\
& \quad\left(h\left(t,\left(p_{1}, q_{1}\right),\left(p_{2}, q_{2}\right)\right),-\left[\partial_{2} g\left(p_{1}, q_{1}\right)\right]^{-1} \partial_{1} g\left(p_{1}, q_{1}\right) h\left(t,\left(p_{1}, q_{1}\right),\left(p_{2}, q_{2}\right)\right)\right),
\end{aligned}
$$

and then define

$$
\Phi=\left.\Psi\right|_{M} \quad \text { and } \quad \Xi=\left.\Upsilon\right|_{\mathbb{R} \times M \times M} .
$$

Since $T_{(p, q)} M$ coincides with the kernel of the differential of $g$ at any $(p, q) \in M$, it can be easily seen that $\Phi(p, q) \in T_{(p, q)} M$ and that $\Xi\left(t,\left(p_{1}, q_{1}\right),\left(p_{2}, q_{2}\right)\right) \in$ $T_{\left(p_{1}, q_{1}\right)} M$, for all $\left(t,\left(p_{1}, q_{1}\right),\left(q_{2}, p_{2}\right)\right) \in \mathbb{R} \times M \times M$. Therefore, the following is a delay differential equation on $M$ :

$$
\dot{\zeta}(t)=a(t) \Phi(\zeta(t))+\lambda \Xi(t, \zeta(t), \zeta(t-r)), \quad \lambda \geq 0 .
$$

Lemma 6.4. Equation (6.9) is equivalent to (1.5), in the sense that $\zeta=(x, y)$ is a solution of (6.9), on an interval $I \subseteq \mathbb{R}$, if and only if so is $(x, y)$ for (1.5). 
Proof. Let $x: J \rightarrow \mathbb{R}^{k}$ and $y: J \rightarrow \mathbb{R}^{s}$ be $C^{1}$ maps defined on an interval $J$ with the property that $t \mapsto \xi(t)=(x(t), y(t))$ is a solution of (6.9). Then, for all $t \in J$,

$$
\dot{x}(t)=f(x(t), y(t))+\lambda h(t, x(t), y(t)),
$$

and, since $(x(t), y(t)) \in M$, we have $g(x(t), y(t))=0$.

Conversely, let $t \mapsto \zeta(t)=(x(t), y(t))$ be a solution of (1.5). Then,

$$
\dot{x}(t)=f(x(t), y(t))+\lambda h(t, x(t), y(t)),
$$

and, differentiating $g(x(t), y(t))=0$ at any $t \in J$, one gets

$$
\partial_{1} g(x(t), y(t)) \dot{x}(t)+\partial_{2} g(x(t), y(t)) \dot{y}(t)=0,
$$

for all $t \in J$. So that

$$
\begin{aligned}
\dot{y}(t)= & -\left(\partial_{2} g(x(t), y(t))\right)^{-1} \partial_{1} g(x(t), y(t)) \dot{x}(t) \\
& =-\left(\partial_{2} g(x(t), y(t))\right)^{-1} \partial_{1} g(x(t), y(t))[f(x(t), y(t))+\lambda h(t, x(t), y(t))] .
\end{aligned}
$$

Thus $\dot{\zeta}(t)=(\dot{x}(t), \dot{y}(t))=\Psi(\zeta(t))+\lambda \Upsilon(t, \zeta(t))$. Notice also that for all $t \in J$, $\zeta(t) \in M$ because $g(x(t), y(t))=0$. So, on account of (6.8), we have $\Psi(\zeta(t))=$ $\Phi(\zeta(t))$ and $\Upsilon(t, \zeta(t))=\Xi(t, \zeta(t))$. Thus,

$$
\dot{\zeta}(t)=\Phi(\zeta(t))+\lambda \Xi(t, \zeta(t)) .
$$

And the assertion is proved.

Using the above lemma, we can combine the results of Theorems 2.4 and 5.1. The map $F: U \rightarrow \mathbb{R}^{k} \times \mathbb{R}^{s}$ introduced in (2.6) becomes, in our case,

$$
F(x, y)=(f(x, y), g(x, y)) .
$$

So, with the notation recalled above, the set of trivial $T$-periodic pairs can be written as $\left\{(0,(\bar{p}, \bar{q})) \in[0, \infty) \times C_{T}(U): F(p, q)=(0,0)\right\}$. Also, as in Section 5 , given $\Omega \subseteq[0, \infty) \times C_{T}(U)$, we denote by $\Omega \cap U$ the subset of $U$ whose points, regarded as constant functions, lie in $\Omega$. Namely,

$$
\Omega \cap U=\{(p, q) \in U:(0,(\bar{p}, \bar{q})) \in \Omega\} .
$$

We finally state and prove the following consequence of Theorems 2.4 and 5.1, which is inspired to [2, Theorem 5.1] and generalizes the main result of [1].

Theorem 6.5. Let $U \subseteq \mathbb{R}^{k} \times \mathbb{R}^{s}$ be open and connected. Let $g: U \rightarrow \mathbb{R}^{s}$, $f: U \rightarrow \mathbb{R}^{k}, a: \mathbb{R} \rightarrow \mathbb{R}$ and $h: \mathbb{R} \times U \rightarrow \mathbb{R}^{k}$ be as above. Let also $F(p, q)=$ $(f(p, q), g(p, q))$. Given $\Omega \subseteq[0, \infty) \times C_{T}(U)$ open, assume that $\operatorname{deg}(F, \Omega \cap U)$ is well-defined and nonzero. Then, there exists a connected set of nontrivial solution pairs of (1.5) whose closure in $\Omega$ is noncompact and meets the set $\{(0,(\bar{p}, \bar{q})) \in$ $\Omega: F(p, q)=(0,0)\}$ of the trivial T-periodic pairs of (1.5). 
Proof. Let $\Phi$ and $\Xi$ be the tangent vector fields defined in (6.8). Let also $\mathcal{O}$ be the open subset of $[0, \infty) \times C_{T}(M)$ given by

$$
\mathcal{O}=\Omega \cap\left([0, \infty) \times C_{T}(M)\right) .
$$

For any $Y \subseteq M$, by $\mathcal{O} \cap Y$ we mean the set of all those points of $Y$ that, regarded as constant functions, lie in $\mathcal{O}$. Using this convention, one has that $\Omega \cap Y=\mathcal{O} \cap Y$ and, in particular, $\Omega \cap M=\mathcal{O} \cap M$. Thus, Theorem 2.4 implies that

$$
|\operatorname{deg}(\Phi, \mathcal{O} \cap M)|=|\operatorname{deg}(\Phi, \Omega \cap M)|=|\operatorname{deg}(F, \Omega \cap U)| \neq 0 .
$$

Theorem 5.1 yields a connected set $\Lambda$ of nontrivial $T$-periodic pairs of (6.9) whose closure in $\mathcal{O}$ is not compact and meets the set

$$
\{(0,(\bar{p}, \bar{q})) \in \mathcal{O}: \Phi(p, q)=(0,0)\}=\{(0, \bar{p}, \bar{q}) \in \Omega: F(p, q)=(0,0)\} .
$$

The equivalence of (6.9) with (1.5) imply that each $(\lambda,(x, y)) \in \Lambda$ is a nontrivial $T$-periodic pair of (1.5) as well. Since $M$ is closed in $U$, any relatively closed subset of $\mathcal{O}$ is relatively closed in $\Omega$ too and vice versa. Thus, the closure of $\Lambda$ in $\mathcal{O}$ coincides with the closure of $\Lambda$ in $\Omega$, and hence $\Lambda$ fulfills the assertion.

\section{REFERENCES}

[1] L. Bisconti, Harmonic solutions to a class of Differential-Algebraic Equations with separated variables, Electron. J. Differential Equations, 2012 (2012), No. 2, 1-15.

[2] A. Calamai and M. Spadini, Branches of forced oscillations for a class of constrained ODEs: a topological approach, NoDEA Nonlinear Differential Equations Appl. 19 (2012), No. 4, 383-399.

[3] J. DugundiJ, Topology, Allyn and Bacon series in advanced mathematics, Allyn and Bacon, Boston, 1966.

[4] M. Furi And M.P. Pera, A continuation principle for periodic solutions of forced motion equations on manifolds and application to bifurcation theory, Pacific J. Math. 160 (1993), 219-244.

[5] M. Furi, M.P. Pera and M. Spadini, The fixed point index of the Poincaré operator on differentiable manifolds, Handbook of topological ixed point theory (R.F. Brown, M. Furi, L. Górniewicz and B. Jiang, eds.), Spinger, 2005.

[6] M. Furi And M. SPADini, On the set of harmonic solutions of periodically perturbed autonomous differential equations on manifolds, Nonlinear Anal. 29 (1997), No. 8, 963970.

[7] _ Periodic perturbations with delay of autonomous differential equations on manifolds, Adv. Nonlinear Stud. 9 (2009), No. 2, 263-276.

[8] V. Guillemin and A. Pollack, Differential Topology, Prentice-Hall Inc., Englewood Cliffs, New Jersey, 1974.

[9] P. Kunkel and V. Mehrmann, Differential-Algebraic Equations: Analysis and Numerical Solutions., EMS Textbooks in Mathematics, 2006.

[10] S. Lang, Differential Manifolds, Addison Wesley Pubbl. Comp. Inc., Reading, Massachusetts, 1972 .

[11] J.W. Milnor, Topology from the differentiable viewpoint, Univ. press of Virginia, Charlottesville, 1965. 
[12] W.M. OLIVA, Functional differential equations on compact manifolds and an approximation theorem, J. Differential Equations 5 (1969), 483-496.

[13] M. SPADINI, Harmonic solutions to perturbations of periodic separated variables ODEs on manifolds, Electron. J. Differential Equations, 2003 (2003), No. 88, 1-11.

[14] _ A note on topological methods for a class of Differential-Algebraic Equations, Nonlinear Anal. 73 (2010), 1065-1076.

Manuscript received September 17, 2013

Luca Bisconti And Marco Spadini

Dipartimento di Sistemi e Informatica

Università degli Studi di Firenze

Via S. Marta 3

50139 Firenze, ITALY

E-mail address: luca.bisconti@unifi.it, marco.spadini@unifi.it 